\title{
Cyclic Demazure modules and positroid varieties
}

\author{
Thomas Lam * \\ Department of Mathematics \\ University of Michigan \\ 530 Church Street \\ Ann Arbor, MI, U.S.A. \\ tfylam@umich.edu
}

Submitted: Dec 14, 2018; Accepted: May 1, 2019; Published: May 17, 2019

(C) The author. Released under the CC BY-ND license (International 4.0).

\begin{abstract}
A positroid variety is an intersection of cyclically rotated Grassmannian Schubert varieties. Each graded piece of the homogeneous coordinate ring of a positroid variety is the intersection of cyclically rotated (rectangular) Demazure modules, which we call the cyclic Demazure module. In this note, we show that the cyclic Demazure module has a canonical basis, and define the cyclic Demazure crystal.
\end{abstract}

Mathematics Subject Classifications: 05E10,14N15

\section{Introduction}

The classical Borel-Weil theorem identifies the global sections $\Gamma\left(G / B, L_{\lambda}\right)$ of a line bundle on a flag variety with the irreducible highest weight representation $V(\lambda)$. When the same line bundle is restricted to a Schubert variety $X_{w}$, the global sections $\Gamma\left(X_{w}, L_{\lambda}\right)$ can be identified with the Demazure module $V_{w}(\lambda)$. In this paper, we study the global sections $\Gamma\left(\Pi_{f}, \mathcal{O}(d)\right)$ of a line bundle on a positroid subvariety $\Pi_{f}$ of the Grassmannian $\operatorname{Gr}(k, n)$.

Positroid varieties (see Section 4) are certain intersections of cyclically rotated Schubert varieties in the Grassmannian. They were introduced in Postnikov's work [Pos] on the totally nonnegative Grassmannian, and subsequently studied in algebro-geometric terms by Knutson-Lam-Speyer [KLS13]. Via [KLS13], the work of Lakshmibai and Littelmann [LaLi] gives a description of the vector space $\Gamma\left(\Pi_{f}, \mathcal{O}(d)\right)$ in terms of standard monomials. In the present work, we give a new description of $\Gamma\left(\Pi_{f}, \mathcal{O}(d)\right)$ that is compatible with the cyclic symmetry of the Grassmannian and its positroid varieties.

\footnotetext{
*Supported by NSF grants DMS-1160726 and DMS-1464693, and by a Von Neumann fellowship at the Institute of Advanced Study.
} 
We define in Section 5 the cyclic Demazure module $V_{f}\left(d \omega_{k}\right)$ as the intersection of cyclically rotated Demazure modules. We show in Theorem 15 that a graded piece of the homogeneous coordinate ring of a positroid variety can be identified with the (dual of the) cyclic Demazure module.

We define the cyclic Demazure crystal $B_{f}\left(d \omega_{k}\right)$ as the intersection of cyclically rotated (via promotion) Demazure crystals. We show in Theorem 14 that $V_{f}\left(d \omega_{k}\right)$ has a basis given by the canonical basis elements indexed by $B_{f}\left(d \omega_{k}\right)$. We obtain the following dichotomy (Theorem 23): a dual canonical basis element either (1) vanishes on $\Pi_{f}$ (if it is outside $B_{f}\left(d \omega_{k}\right)$ ), or $(2)$ it takes strictly positive values on the totally positive part $\Pi_{f,>0}$ (if it is inside $\left.B_{f}\left(d \omega_{k}\right)\right)$.

Our approach is based on the key observation (Theorem 1(iv)) that the dual canonical basis of the Grassmannian is invariant under signed cyclic rotation. This relies heavily on the work of Rhoades [Rho]. In Theorem 1(ii), we show that dual canonical basis in degree two is identical to the Temperley-Lieb invariants of [Lam14], which are defined in a combinatorially explicit manner.

Our work was initially motivated by the budding theory of Grassmann polytopes, and many of the results here were announced initially in [Lam16]. However, the results herein were so simple and clean, we felt that they deserved a short and separate exposition. We plan to pursue our intended applications in other work. In Section 6, we also indicate some further directions of study.

Acknowledgements. We are grateful to Nima Arkani-Hamed, Allen Knutson, Alex Postnikov, Mark Shimozono, and David Speyer for conversations over the years related to this work. We thank the referee for a number of helpful suggestions.

\section{The dual canonical basis of the Grassmannian}

Let $[n]:=\{1,2, \ldots, n\}$ and $\left(\begin{array}{c}{[n]} \\ k\end{array}\right)$ denote the collection of $k$-element subsets of $[n]$.

\subsection{The Grassmannian and its homogeneous coordinate ring}

Let $\operatorname{Gr}(k, n)$ denote the Grassmannian of $k$-planes in $\mathbb{C}^{n}$ and let $\hat{\operatorname{Gr}}(k, n)$ denote the affine cone over the Grassmannian. A point $X \in \hat{\mathrm{G}} \mathrm{r}(k, n)$ is determined by a set $\Delta_{I}(X)$ of Plücker coordinates satisfying the Plücker relations, where $I \in\left(\begin{array}{c}{[n]} \\ k\end{array}\right)$ (see [Lam16, Section $3]$ ). We allow the possibility that all $\Delta_{I}(X)$ are simultaneously zero. The Grassmannian $\operatorname{Gr}(k, n)$ is the quotient of $\hat{\operatorname{Gr}}(k, n)-\{0\}$ by the equivalence relation of simultaneously scaling all Plücker coordinates by the same scalar.

Let $\hat{\operatorname{Gr}}(k, n)_{\geqslant 0}$ denote the cone over the totally nonnegative Grassmannian: it consists of points $X \in \hat{\operatorname{Gr}}(k, n)_{\geqslant 0}$ where $\Delta_{I}(X) \geqslant 0$ for all $I$. The totally nonnegative Grassmannian $\operatorname{Gr}(k, n)_{\geqslant 0}$ [Pos] is the image of $\hat{\operatorname{Gr}}(k, n)_{\geqslant 0}$ in $\operatorname{Gr}(k, n)$.

Let $R(k, n)$ denote the coordinate ring of $\hat{\operatorname{Gr}}(k, n)$, or equivalently, the homogeneous coordinate ring of $\operatorname{Gr}(k, n)$. Thus,

$$
R(k, n)=\mathbb{C}\left[\Delta_{I}\right] /(\text { Plücker relations })
$$


is a graded ring where the degree of $\Delta_{I}$ is taken to be 1 . For example,

$$
R(2,4)=\mathbb{C}\left[\Delta_{12}, \Delta_{13}, \Delta_{14}, \Delta_{23}, \Delta_{24}, \Delta_{34}\right] /\left(\Delta_{13} \Delta_{24}-\Delta_{12} \Delta_{34}-\Delta_{14} \Delta_{23}\right) .
$$

We also note that $R(k, n)$ is a unique factorization domain. We let $R(k, n)_{d}$ denote the $d$ th graded piece of $R(k, n)$, spanned by monomials $\Delta_{I_{1}} \Delta_{I_{2}} \cdots \Delta_{I_{d}}$. We begin by reviewing the classical description of $R(k, n)_{d}$ in representation theoretic terms.

\section{$2.2 \quad$ Highest weight representations}

A partition $\lambda=\left(\lambda_{1} \geqslant \lambda_{2} \geqslant \cdots \geqslant \lambda_{\ell}>0\right)$ is a weakly decreasing sequence of positive integers. We say that $\lambda=\left(\lambda_{1} \geqslant \lambda_{2} \geqslant \cdots \geqslant \lambda_{\ell}>0\right)$ has $\ell$ parts and size $|\lambda|=$ $\lambda_{1}+\lambda_{2}+\cdots+\lambda_{\ell}$. We have the following dominance order on partitions: $\lambda \geqslant \mu$ if and only if $|\lambda|=|\mu|$ and $\lambda_{1} \geqslant \mu_{1}, \lambda_{1}+\lambda_{2} \geqslant \mu_{1}+\mu_{2}$, and so on.

For a partition $\lambda$ with at most $n$ parts, we have an irreducible, finite-dimensional representation $V(\lambda)$ of $\mathrm{GL}(n)$ with highest weight $\lambda$. We state some basic facts concerning $V(\lambda)$.

The Young diagram of $\lambda$ is the collection of boxes in the plane with $\lambda_{1}$ boxes in the 1st row, $\lambda_{2}$ boxes in the 2 nd row, and so on, where all boxes are upper-left justified. A semistandard tableaux $T$ of shape $\lambda$ is a filling of the Young diagram of $\lambda$ by the numbers $1,2, \ldots, n$ so that each row is weakly-increasing, and each column is strictly increasing. The weight $\operatorname{wt}(T)$ of a tableau $T$ is the composition $\left(\alpha_{1}, \alpha_{2}, \ldots, \alpha_{n}\right)$ where $\alpha_{i}$ is equal to the number of $i$-s in $T$. For example,

$$
\begin{array}{|l|l|l|l|l|}
\hline 1 & 1 & 3 & 4 & 4 \\
\cline { 1 - 3 } 2 & 3 & 4 & 5 & \multicolumn{1}{|c}{} \\
\cline { 1 - 3 } 4 & 4 & \multicolumn{3}{|c}{} \\
\cline { 1 - 2 } & \multicolumn{4}{|c}{}
\end{array}
$$

is a semistandard tableau with shape $(5,4,2)$ with weight $(2,1,2,5,1)$. Let $B(\lambda)$ denote the set of semistandard tableaux of shape $\lambda$. (Note that this set depends on $n$, which is suppressed from the notation.) The dimension $\operatorname{dim}(V(\lambda))$ is equal to the cardinality of $B(\lambda)$. A vector $v$ in a $\operatorname{GL}(n)$-representation $V$ is called a weight vector with weight $\alpha=\left(\alpha_{1}, \alpha_{2}, \ldots, \alpha_{n}\right)$ if the diagonal matrix $\operatorname{diag}\left(x_{1}, x_{2}, \ldots, x_{n}\right)$ sends $v$ to $\left(x_{1}^{\alpha_{1}} x_{2}^{\alpha_{2}} \cdots x_{n}^{\alpha_{n}}\right) v$.

Lusztig [Lus90] and Kashiwara [Kas93a] have constructed a canonical basis, or global basis of the $U_{q}\left(\mathfrak{s l}_{n}\right)$-module $V_{q}(\lambda)$. We shall only use the evaluation of this basis at $q=1$. After picking a highest weight vector $v_{+}$for $V(\lambda)$, this is a distinguished basis $\{G(T) \mid T \in B(\lambda)\}$ of $V(\lambda)$ such that each $G(T)$ is a weight vector with weight $w t(T)$ (see [Lus90, Section 8.11]).

We shall also let $\{H(T) \mid T \in B(\lambda)\}$ denote the dual basis of $V(\lambda)^{*}$, called the dual canonical basis. Let $(\cdot, \cdot)$ denote the unique nondegenerate symmetric bilinear form on $V(\lambda)$ satisfying $\left(v_{+}, v_{+}\right)=1$, and $(x \cdot v, u)=\left(v, x^{T} \cdot u\right)$ where $x \in \mathfrak{g l}_{n}$ and $x^{T}$ denotes the transpose. We may identify $V(\lambda)$ with $V(\lambda)^{*}$ via $(\cdot, \cdot)$, and $H(T)$ becomes a basis of $V(\lambda)$. 


\subsection{Crystals}

The set $B(\lambda)$ has the structure of a crystal graph. We will only need the operations

$$
\tilde{e}_{i}: B(\lambda) \rightarrow B(\lambda) \cup\{0\}
$$

and

$$
\tilde{f}_{i}: B(\lambda) \rightarrow B(\lambda) \cup\{0\}
$$

for $i=1,2, \ldots, n-1$. Let $T \in B(\lambda)$. The rowword $\operatorname{row}(T)$ of $T$ is obtained by reading the rows of $T$ from left to right, starting from the bottom row.

For a fixed $i \in\{1,2, \ldots, n-1\}$, we think of each occurrence of $i$ in $\operatorname{row}(T)$ to be a closed parenthesis ")" and each occurrence of $i+1$ in $\operatorname{row}(T)$ to be an open parenthesis "(". We then pair these parentheses as usual until no pairing can be done. We are left with a sequence that looks like ")) ))((". The operation $\tilde{f}_{i}$ changes the $i$ corresponding to the rightmost unpaired ")" into a $i+1$. The operation $\tilde{e}_{i}$ changes the $i+1$ corresponding to the leftmost unpaired "(" into a $i$. The result will be the rowword of a unique tableau $\tilde{e}_{i}(T)$ or $\tilde{f}_{i}(T)$ of shape $\lambda$. If there is no such ")" (resp. "("), then $\tilde{f}_{i}(T)\left(\operatorname{resp} . \tilde{e}_{i}(T)\right)$ is defined to be 0 .

\subsection{Kirillov-Reshetikhin crystals}

Let $\omega_{k}=(1,1, \ldots, 1)$ be the partition with $k 1$ 's. Then $V\left(\omega_{k}\right)$ is isomorphic to the $k$ exterior power $\Lambda^{k}\left(\mathbb{C}^{n}\right)$ of the standard representation $\mathbb{C}^{n}$ of $\mathrm{GL}(n)$ and the canonical basis of $V\left(\omega_{k}\right)$ is simply the basis $\left\{e_{i_{1}} \wedge e_{i_{2}} \wedge \cdots \wedge e_{i_{k}}\right\}$. For an integer $d \geqslant 1$, the representation $V\left(d \omega_{k}\right)$ for a rectangular partition has very special properties. The set $B\left(d \omega_{k}\right)$ is the set of semistandard Young tableaux with $k$ rows and $d$ columns. For example,

\begin{tabular}{|l|l|l|l|l|}
\hline 1 & 1 & 3 & 4 & 4 \\
\hline 2 & 3 & 4 & 5 & 5 \\
\hline 4 & 4 & 6 & 6 & 6 \\
\hline
\end{tabular}

belongs to $B\left(5 \omega_{3}\right)$.

The crystal $B\left(d \omega_{k}\right)$ has an additional operation called promotion, which is a bijection $\chi: B\left(d \omega_{k}\right) \rightarrow B\left(d \omega_{k}\right)$ such that $\chi^{n}=1$ and $\chi \circ \tilde{e}_{i}=\tilde{e}_{i+1} \circ \chi\left(\operatorname{resp} . \chi \circ \tilde{f}_{i}=\tilde{f}_{i+1} \circ \chi\right)$ (see [Shi]). This defines extra operations $\tilde{e}_{0}$ and $\tilde{f}_{0}$ on $B\left(d \omega_{k}\right)$, and the index $i$ of $\tilde{e}_{i}$ (resp. $\tilde{f}_{i}$ ) will always be considered modulo $n$. Together these structures form part of the affine crystal structure of $B\left(d \omega_{k}\right)$, which in this case is a Kirillov-Reshetikhin crystal, see [Shi, HKOTT].

Promotion is defined as follows: first remove all occurrences of the letter $n$ in $T$. Then slide the boxes to the bottom right of the rectangle, always keeping the rows weaklyincreasing and columns strictly-increasing. Once all slides are complete, add one to all remaining letters and fill the empty boxes with the letter 1 to obtain $\chi(T)$. For example,

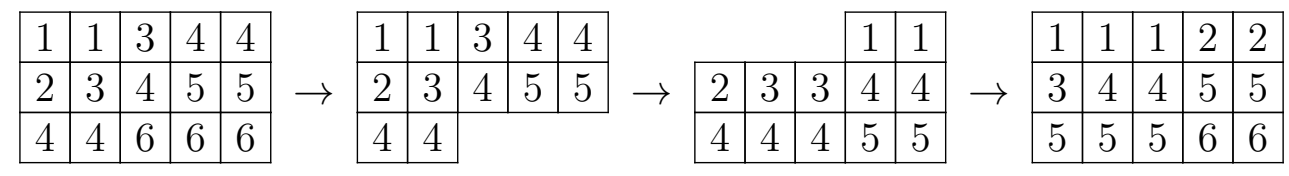




\subsection{The dual canonical basis of the Grassmannian}

By the classical Borel-Weil theorem, the degree $d$ component $R(k, n)_{d}$ of the graded ring $R(k, n)$ is canonically isomorphic, as a $\mathrm{GL}(n)$-representation, to the dual $V\left(d \omega_{k}\right)^{*}$ of the highest weight representation $V\left(d \omega_{k}\right)$.

Let $\chi: \mathbb{C}^{n} \rightarrow \mathbb{C}^{n}$ denote the (signed) cyclic rotation linear map given by sending $e_{i}$ to $e_{i+1}$ for $i=1,2, \ldots, n-1$ and sending $e_{n}$ to $(-1)^{k-1} e_{1}$. Since $\chi \in \operatorname{GL}(n)$, we obtain a cyclic rotation map $\chi: V\left(d \omega_{k}\right) \rightarrow V\left(d \omega_{k}\right)$. Abusing notation, also define $\chi:[n] \rightarrow[n]$ by the formula $\chi(i)=i+1 \bmod n$. Then $\chi$ also acts on subsets of $[n]$.

We have an induced rotation map $\chi: \operatorname{Gr}(k, n) \rightarrow \operatorname{Gr}(k, n)$. Define $\chi^{*}: R(k, n) \rightarrow$ $R(k, n)$ to be the pullback ring homomorphism induced by $\chi^{-1}: \operatorname{Gr}(k, n) \rightarrow \operatorname{Gr}(k, n)$. It is uniquely determined by $\chi^{*}\left(\Delta_{I}\right)=\Delta_{\chi(I)}$.

Theorem 1. The vector space $R(k, n)_{d}$ has a dual canonical basis $\left\{H(T) \mid T \in B\left(d \omega_{k}\right)\right\}$ with the following properties:

(i) For $d=1$, we have $H(T)=\Delta_{I}$, where $I$ is the set of entries in the one-column tableau $T$.

(ii) For $d=2$, the set $\left\{H(T) \mid T \in B\left(2 \omega_{k}\right)\right\}$ is exactly the set of Temperley-Lieb invariants $\left\{\Delta_{(\tau, T)} \mid(\tau, T) \in \mathcal{A}_{k, n}\right\}$ of Section 3.

(iii) For any $T \in B\left(d \omega_{k}\right)$, the function $H(T)$ is a nonnegative function on $\hat{\operatorname{Gr}}(k, n)_{\geqslant 0}$.

(iv) For any $T \in B\left(d \omega_{k}\right)$, we have $\chi^{*}(H(T))=H(\chi(T))$.

Theorem 1(i) is well-known. Theorem 1(ii) will be discussed in Section 3. Theorem 1(iii) is due to Lusztig [Lus94]. We deduce Theorem 1(iv) from a result of Rhoades [Rho] in the next subsection.

Already for $d=3$, the canonical basis of $V\left(3 \omega_{k}\right)$ is combinatorially obscure to us. In [Lam14], we studied the closely related web basis in combinatorial terms.

\subsection{Cyclicity of canonical basis}

Theorem 2. We have $\chi(G(T))=G(\chi(T))$ and $\chi^{*}(H(T))=H(\chi(T))$.

Proof. Let $\tilde{\chi}$ denote the unsigned cyclic rotation map that sends $e_{i}$ to $e_{i+1} \bmod n$. We first show that $\tilde{\chi}(G(T))= \pm G(\chi(T))$.

Let $\mathcal{A}(n)$ denote the coordinate ring of $n \times n$ matrices, so that $\mathcal{A}(n)=\mathbb{C}\left[x_{i j} \mid i, j \in[n]\right]$. The ring $\mathcal{A}(n)$ has a dual canonical basis $b_{P, Q}$ labeled by pairs of semistandard tableaux $P, Q$ of the same shape and entries bounded by $n$. The cyclic rotation $\tilde{\chi}$ acts on $\mathcal{A}(n)$ by sending the matrix entry $x_{i j}$ to $x_{i+1, j}$ where indices are taken modulo $n$. Equivalently, thinking of $\mathcal{A}(n)$ as the space of polynomial functions on $\operatorname{End}\left(\mathbb{C}^{n}\right) \cong \mathfrak{g l}_{n}$, we have

$$
(\tilde{\chi} \cdot f)(g)=f\left(\tilde{\chi}^{-1} g\right)
$$


for $f \in \mathcal{A}(n)$ and $g \in \operatorname{End}\left(\mathbb{C}^{n}\right)$. In [Rho, Proposition 5.5], Rhoades shows that when $P, Q$ have rectangular shape, we have

$$
\tilde{\chi} \cdot b_{P, Q}= \pm b_{\chi(P), Q}+\text { other terms }
$$

where the other terms belong to the span of the dual canonical basis indexed by shapes different to the shape of $P, Q$.

The basis studied in [Rho] is connected to the canonical bases of the highest weight representations $V\left(d \omega_{k}\right)$ via the works of Skandera [Ska] and Du [Du92]. Specifically, let $\mu^{*}: V\left(d \omega_{k}\right) \rightarrow V\left(d \omega_{k}\right) \otimes \mathcal{A}(n)$ be the coaction map dual to the action map $\mu$ : $V\left(d \omega_{k}\right)^{*} \times \operatorname{End}\left(\mathbb{C}^{n}\right) \rightarrow V\left(d \omega_{k}\right)^{*}$. Du shows that the coaction sends the highest weight vector $v_{+}$of $V\left(d \omega_{k}\right)$ to the sum

$$
\mu^{*}\left(v_{+}\right)=\sum_{b_{P, Q} \in T(d, k)} G(P) \otimes b_{P, Q}
$$

where $T(d, k)$ is some subset of the dual canonical basis of $\mathcal{A}(n)$ and $G(P)$ belongs to the canonical basis of $V\left(d \omega_{k}\right)$. (The coincidence of Du's basis with Lusztig's is shown in [Du95].) The coaction and action are related by the formula $\mu^{*}(v)(w, g)=\langle\mu(w, g), v\rangle$, where $\langle\cdot, \cdot\rangle$ denotes the natural pairing $V\left(d \omega_{k}\right)^{*} \otimes V\left(d \omega_{k}\right) \rightarrow \mathbb{C}$, and $w \in V\left(d \omega_{k}\right)^{*}$ and $g \in \operatorname{End}\left(\mathbb{C}^{n}\right)$. For any $v \in V\left(d \omega_{k}\right), w \in V\left(d \omega_{k}\right)^{*}$, and $g \in \operatorname{End}\left(\mathbb{C}^{n}\right)$, using (1), we have

$$
\left((\tilde{\chi} \otimes \tilde{\chi}) \mu^{*}(v)\right)(w, g)=\left\langle\mu\left(w, \tilde{\chi}^{-1} g\right), \tilde{\chi} v\right\rangle=\langle\tilde{\chi} \mu(w, g), \tilde{\chi} v\rangle=\langle\mu(w, g), v\rangle=\mu^{*}(v)(w, g) .
$$

Thus, $(\tilde{\chi} \otimes \tilde{\chi}) \mu^{*}(v)=\mu^{*}(v)$ and in particular, we have $(\tilde{\chi} \otimes \tilde{\chi}) \mu^{*}\left(v_{+}\right)=\mu^{*}\left(v_{+}\right)$. It follows that

$$
\mu^{*}\left(v_{+}\right)=\sum_{b_{P, Q} \in T(m, k)} \tilde{\chi}(G(P)) \otimes \tilde{\chi} \cdot b_{P, Q}
$$

The set $T(d, k)$ consists of all $b_{P, Q}$ where $Q$ is some fixed semistandard tableaux of rectangular shape $d^{k}$. It follows that $\tilde{\chi} \cdot b_{P, Q} \in T(d, k)$, and in (3) all the "other terms" from (2) cancel out. We conclude that $\tilde{\chi}(G(P))= \pm G(\chi(P))$.

It follows easily from the fact that $G(P)$ is a weight-vector that $\chi(G(P))= \pm G(\chi(P))$ and by duality we have $\chi^{*}(H(P))= \pm H(\chi(P))$. Now $\chi\left(\operatorname{Gr}(k, n)_{\geqslant 0}\right)=\operatorname{Gr}(k, n)_{\geqslant 0}$, so it follows from Theorem 1(iii) that we must have $\chi^{*}(H(P))=H(\chi(P))$. (Note that $H(P)$ cannot be identically 0 on $\operatorname{Gr}(k, n)_{\geqslant 0}$ because the latter is Zariski-dense in $\operatorname{Gr}(k, n)$.)

\section{Temperley-Lieb immanants}

\subsection{Dual canonical basis for $R(k, n)_{2}$}

A $(k, n)$-partial noncrossing pairing $(\tau, T)$ consists of a noncrossing pairing $\tau$ of a subset $S \subset[n]$ together with a subset $T \subset[n] \backslash S$ of marked vertices, satisfying $2|T|+|S|=k$. In [Lam14] we constructed a basis $\left\{\Delta_{(\tau, T)}\right\}$ for $R(k, n)_{2}$ labeled by $(k, n)$-partial noncrossing pairings $(\tau, T)$. Here is an example of a $(4,8)$-partial non-crossing pairing, where the vertex 6 is marked: 


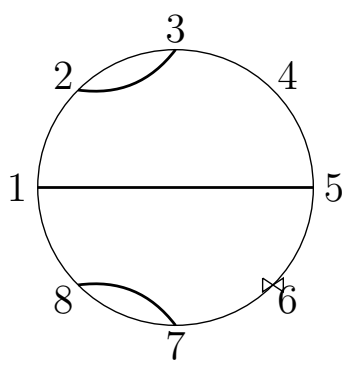

Let $(I, J) \in\left(\begin{array}{c}{[n]} \\ k\end{array}\right)^{2}$. We say that that $(\tau, T)$ is compatible with $(I, J)$ if (a) $I \cap J=T$, and (b) each pair of matched boundary vertices in $\tau$ contains one element of $I$ and one element of $J$. Let $\mathcal{C}(I, J)$ denote the set of $(k, n)$-partial noncrossing pairings that are compatible with $(I, J)$. We have the following identity [Lam14] which determines the elements $\Delta_{(\tau, T)} \in R(k, n)_{2}$ uniquely.

Theorem 3. Let $I, J \in\left(\begin{array}{c}{[n]} \\ k\end{array}\right)$. Then

$$
\Delta_{I} \Delta_{J}=\sum_{(\tau, T) \in \mathcal{C}(I, J)} \Delta_{(\tau, T)}
$$

Define a bijection $\theta: \mathcal{A}_{k, n} \rightarrow B\left(2 \omega_{k}\right)$ as follows. Given $(\tau, T)$, the tableau $\theta(\tau, T)$ has columns $I, J$, where $I \cap J=T$, and for each strand $(a, b) \in \tau$ with $a<b$, we have $a \in I$ and $b \in J$. We next describe the inverse $\theta^{-1}: B\left(2 \omega_{k}\right) \rightarrow \mathcal{A}_{k, n}$. Given a two-column tableau with columns $I$ and $J$, we construct $\theta^{-1}(I, J)=(\tau, T)=(\tau(I, J), T)$. First, set $T=I \cap J$. Next, we think of $\tau$ as partial noncrossing matching of $n$ points $1,2, \ldots, n$ arranged from left to right on a line. Mark the points of $I \backslash J$ as "left endpoints" and the points of $J \backslash I$ as "right endpoints". Draw a strand connecting each left endpoint $p$ to the leftmost right endpoint $q$ such that between $p$ and $q$ there is an equal number of left and right endpoints. The tableau condition for $(I, J)$ guarantees that for each $p \in I \backslash J$ there exists such a $q \in J \backslash I$. The collection of these strands gives $\tau=\tau(I, J)$.

We now prove Theorem 1(ii).

Proposition 4. We have $\Delta_{(\tau, T)}=H(\theta(\tau, T))$. Thus Temperley-Lieb immanants are the dual canonical basis of $V\left(2 \omega_{k}\right)$.

Proof. We deduce the proposition from setting $q=1$ in work of Brundan [Bru] and ChengWang-Zhang [CWZ]. In [CWZ, Section 4], the dual canonical basis for $V\left(\omega_{k}\right) \otimes V\left(\omega_{k}\right)$ is constructed for any $k$. The dual canonical basis elements are denoted $\mathcal{L}_{f}$ in [CWZ]; we shall write them as $\mathcal{L}_{I, J}$ where $I, J$ are $k$-element subsets of $[n]$ (note that [CWZ] are working with $n=\infty)$. The standard basis of $V\left(\omega_{k}\right) \otimes V\left(\omega_{k}\right)$ will be denoted by $\mathcal{K}_{I, J}$.

By [Bru, Theorem 26], there is a linear map $\xi: V\left(\omega_{k}\right) \otimes V\left(\omega_{k}\right) \rightarrow V\left(2 \omega_{k}\right)$ which sends $\mathcal{L}_{A, B}$ to $H(T)$ if $A, B$ are the two columns of a semistandard tableau $T$ of shape $2^{k}$, and to 0 otherwise. In our notation, the map $\xi$ also sends the standard basis element $\mathcal{K}_{I, J}$ to the monomial $\Delta_{I} \Delta_{J}$. By Theorem 3, it thus suffices to show that for a 2-column tableau $T$ with columns $A, B$, that the coefficient of $\mathcal{L}_{T}:=\mathcal{L}_{A, B}$ in $\mathcal{K}_{I, J}$ is equal to 1 or 0 depending on whether $\theta^{-1}(T)$ is compatible with $(I, J)$ or not. 
Let $A, B$ be two $k$-element subsets of $[n]$. Cheng-Wang-Zhang [CWZ] define a set of pairs $\Sigma_{A, B}^{-}=\{(i, j)\}$ (denoted $\Sigma_{f}^{-}$in [CWZ]). First, let $\mathcal{A}_{A, B}$ be the set of ordered pairs $(i, j)$, where $i \in A \backslash B, j \in B \backslash A$, and $i<j$. Recursively define $\Sigma_{A, B}^{-, r}$ for $r \geqslant 1$ by

$$
\Sigma_{A, B}^{-, r}:=\left\{(i, j) \in \mathcal{A}_{A, B} \mid j-i=r \text { and } i, j \text { do not appear in } \bigcup_{1 \leqslant \ell<r} \Sigma_{A, B}^{-, \ell}\right\} .
$$

We set $\Sigma_{A, B}^{-}:=\bigcup_{r \geqslant 1} \Sigma_{A, B}^{-, r}$. It is then shown in [CWZ, Corollary 4.18] that the coefficient of $\mathcal{L}_{A, B}$ in $\mathcal{K}_{I, J}$ is equal to 1 if $(I, J)$ can be obtained from $(A, B)$ by swapping the pairs in some subset $\Sigma \subseteq \Sigma_{A, B}^{-}$, and equal to 0 otherwise.

Now, suppose that $A, B$ are the two columns of a semistandard tableaux $T$ of shape $2^{k}$. It is then easy to check that $\Sigma_{A, B}^{-}$is exactly the set of strands of the non-crossing matching of $\theta^{-1}(A, B)$. This completes the proof.

\subsection{Explicit formula for Temperley-Lieb invariants}

Call a pair $(I, J)$ standard if $I$ and $J$ form the two columns of a semistandard tableaux. Recall that $\theta^{-1}(I, J)=(\tau(I, J), T=I \cap J)$ is a partial noncrossing matching.

We shall need to consider pairs $(\mathbf{I}, J)$ where $\mathbf{I}$ is an ordered sequence $\left(i_{1}, i_{2}, \ldots, i_{k}\right)$ of distinct integers in $[n]$. Let $\overline{\mathbf{I}} \in\left(\begin{array}{c}{[n]} \\ k\end{array}\right)$ denote the $k$-element subset consisting of the elements of the sequence $\mathbf{I}$. We say that $(\mathbf{I}, J)$ is standard if $(\overline{\mathbf{I}}, J)$ is. We also have a matching $\tau(\mathbf{I}, J):=\tau(\overline{\mathbf{I}}, J)$. We write $\left(\mathbf{I}=\left(i_{1}, \ldots, i_{a}, \ldots, i_{k}\right), J\right) \rightarrow_{a}\left(\mathbf{I}^{\prime}=\left(i_{1}, \ldots, j, \ldots, i_{k}\right), J^{\prime}=\right.$ $\left.J \backslash\{j\} \cup\left\{i_{a}\right\}\right)$ where $\left(i_{a}, j\right) \in \tau(\mathbf{I}, J)$ and if $\left(\mathbf{I}^{\prime}, J^{\prime}\right)$ is a standard pair.

A legal path $P$ of length $|P|=r$ between $(I, J)$ and $(K, L)$ is a sequence

$$
\left(\mathbf{I}_{0}, J\right) \rightarrow_{a_{1}}\left(\mathbf{I}_{1}, J_{1}\right) \rightarrow_{a_{2}} \cdots \rightarrow_{a_{r}}\left(\mathbf{I}_{r}, J_{r}\right)
$$

where $\mathbf{I}_{0}$ is equal to $I$ arranged in order, $\overline{\mathbf{I}}_{r}=K$, and $a_{1} \geqslant a_{2} \geqslant \cdots \geqslant a_{r}$. Note that $I \cup J=K \cup L$ as multisets whenever a legal path exists.

The following result can be deduced from [CWZ]. We give an independent proof.

Theorem 5. We have

$$
\Delta_{(\tau, T)}=\sum_{(I, J)}\left(\sum_{P}(-1)^{|P|}\right) \Delta_{I} \Delta_{J}
$$

where the first summation is over all standard pairs $(I, J)$ and the second summation is over legal paths from $(I, J)$ to $\theta(\tau, T)$.

Example 6. Let $k=3$ and $n=6$. Then the standard pairs $(I, J)$ with $I \cup J=[6]$ are

$$
\text { (123, 456), (124, 356), (134, 256), (125, 346), (135, 246). }
$$

\footnotetext{
${ }^{1}$ The parameters $m$ and $n$ in [CWZ] are both equal to $k$ for us.
} 
The transition matrix from $\left\{\Delta_{I} \Delta_{J}\right\}$ to $\left\{\Delta_{\theta^{-1}(I, J)}\right\}$ and its inverse are

$$
\left[\begin{array}{ccccc}
1 & 1 & 0 & 0 & 1 \\
0 & 1 & 1 & 1 & 1 \\
0 & 0 & 1 & 0 & 1 \\
0 & 0 & 0 & 1 & 1 \\
0 & 0 & 0 & 0 & 1
\end{array}\right] \quad \text { and } \quad\left[\begin{array}{ccccc}
1 & -1 & 1 & 1 & -2 \\
0 & 1 & -1 & -1 & 1 \\
0 & 0 & 1 & 0 & -1 \\
0 & 0 & 0 & 1 & -1 \\
0 & 0 & 0 & 0 & 1
\end{array}\right]
$$

respectively. Reading the last column of the right matrix, we get

$$
\Delta_{\theta^{-1}(135,246)}=\Delta_{135} \Delta_{246}-\Delta_{125} \Delta_{346}-\Delta_{134} \Delta_{256}+\Delta_{124} \Delta_{356}-2 \Delta_{123} \Delta_{456} .
$$

The term $2 \Delta_{123} \Delta_{456}$ arises from the two legal paths

$$
\begin{gathered}
((1,2,3),\{456\}) \rightarrow_{2}((1,5,3),\{246\}) \\
((1,2,3),\{456\}) \rightarrow_{3}((1,2,4),\{356\}) \rightarrow_{3}((1,2,5),\{346\}) \rightarrow_{2}((1,3,5),\{246\}) .
\end{gathered}
$$

Note that the path

$$
((1,2,3),\{456\}) \rightarrow_{3}((1,2,4),\{356\}) \rightarrow_{2}((1,3,4),\{256\}) \rightarrow_{3}((1,3,5),\{246\})
$$

is not legal, because the sequence $3,2,3$ is not weakly decreasing.

If $(i<j),(r<s) \in \tau$ are two strands of a noncrossing matching, we say that $(i, j)$ is nested under $(r, s)$ if $(r<i<j<s)$. We say that $(i, j)$ is nested immediately under $(r, s)$ if, in addition, there is no strand $(p, q)$ such that $(i, j)$ is nested under $(p, q)$, and $(p, q)$ is nested under $(r, s)$.

Lemma 7. Suppose that $(\mathbf{I}, J) \rightarrow_{a}\left(\mathbf{I}^{\prime}, J^{\prime}\right)$, where $\left(i_{a}, j\right)$ is the strand swapped. Then there is a unique $\left(i_{b}, \tilde{j}\right) \in \tau(\mathbf{I}, J)$ such that $\left(i_{a}, j\right)$ is nested immediately under $\left(i_{b}, \tilde{j}\right)$. Furthermore, $\tau\left(\mathbf{I}^{\prime}, J^{\prime}\right)$ is obtained from $\tau(\mathbf{I}, J)$ by replacing the two strands $\left(i_{a}, j\right)$ and $\left(i_{b}, \tilde{j}\right)$ by the two strands $\left(i_{b}, i_{a}\right)$ and $(j, \tilde{j})$.

Proof. If $\left(i_{a}, j\right)$ is not nested under any other strand, then $\left|\overline{\mathbf{I}} \cap\left[1, i_{a}-1\right]\right|=\left|J \cap\left[1, i_{a}-1\right]\right|$. It follows that swapping $i_{a}$ with $j$ in $(\overline{\mathbf{I}}, J)$ cannot give a standard pair. This gives the first statement. It is easy to see that replacing $\left(i_{a}, j\right)$ and $\left(i_{b}, \tilde{j}\right)$ by the two strands $\left(i_{b}, i_{a}\right)$ and $(j, \tilde{j})$ does indeed give a noncrossing matching, and the second statement follows.

Lemma 8. Suppose that we have a legal path ending at $\left(\mathbf{I}=\left(i_{1}, i_{2}, \ldots, i_{k}\right), J\right)$. Suppose that $a<b$ and that $\left(i_{a}, j\right)$ and $\left(i_{b}, \tilde{j}\right)$ are both in $\tau(\mathbf{I}, J)$. Then $\left(i_{a}, j\right)$ is never nested under $\left(i_{b}, \tilde{j}\right)$.

Proof. Let $P=\left(\mathbf{I}_{0}, J\right) \rightarrow_{a_{1}}\left(\mathbf{I}_{1}, J_{1}\right) \rightarrow_{a_{2}} \cdots \rightarrow_{a_{r}}\left(\mathbf{I}_{r}, J_{r}\right)$ be a legal path ending at $\left(\mathbf{I}_{r}, J_{r}\right)=\left(\mathbf{I}=\left(i_{1}, i_{2}, \ldots, i_{k}\right), J\right)$, and suppose $a<b$. We proceed by induction on $r$. If $r=0$, the claim is clear. If $i_{a}<i_{b}$, the claim is clear. Thus we may assume that $i_{a}>i_{b}$ and $a_{r} \leqslant a$. If $a_{r}<a$, then by induction and Lemma 7 , the last swap $\rightarrow_{a_{r}}\left(\mathbf{I}_{r}, J_{r}\right)$ does not affect the strands $\left(i_{a}, j\right)$ and $\left(i_{b}, \tilde{j}\right)$ incident to $i_{a}$ and $i_{b}$.

Finally, suppose that $a_{r}=a$, and let $\left(\mathbf{I}^{\prime}, J^{\prime}\right)=\left(\mathbf{I}_{r-1}, J_{r-1}\right)$. There are two cases: $i_{a}^{\prime}<i_{b}^{\prime}$ and $i_{a}^{\prime}>i_{b}^{\prime}$. In the first case, the claim follows from Lemma 7 , and in the second case, the claim follows from the inductive assumption and Lemma 7. 
Proof of Theorem 5. Any $i \in T$ is present in both $I$ and $J$ for all terms on the RHS. Thus it suffices to prove the statement assuming that $T=\emptyset$ and $\tau$ is a complete noncrossing matching on $[n]=[2 k]$. Henceforth, we make this assumption; thus we restrict to standard pairs $(I, J)$ using each element in $[2 k]$ exactly once. Restricting Theorem 3 to these standard pairs, we must show that (4) and (5) give inverse matrices.

Define a partial order $\leqslant$ on standard pairs by $(I, J) \leqslant(C, D)$ if $i_{r} \leqslant c_{r}$ for $r=$ $1,2, \ldots, k$, where $I=\left\{i_{1}<i_{2}<\cdots<i_{k}\right\}$ and $C=\left\{c_{1}<c_{2}<\cdots<c_{k}\right\}$. A legal path from $(I, J)$ to $(C, D)$ can exist only if $(I, J) \leqslant(C, D)$. Also $\theta^{-1}(C, D)$ is compatible with $(K, L)$ only if $(C, D) \leqslant(K, L)$. The transition matrices from (4) and (5) are triangular with respect to this partial order. Thus let $(I, J) \leqslant(K, L)$ be given. We must show that

$$
\sum_{(C, D)} \sum_{P}(-1)^{|P|}= \begin{cases}1 & \text { if }(I, J)=(K, L), \\ 0 & \text { if }(I, J)<(K, L),\end{cases}
$$

where the first summation is over all standard pairs $(C, D)$ such that $\theta^{-1}(C, D) \in \mathcal{C}(K, L)$, and the second summation is over all legal paths $P$ from $(I, J)$ to $(C, D)$. The statement is clear when $(I, J)=(K, L)$.

Suppose $(I, J)<(K, L)$. We provide a sign-reversing involution $\iota$ on the terms in (6). If $\tau(C, D)$ is compatible with $(K, L)$ we can obtain $(K, L)$ from $(C, D)$ by swapping some (uniquely determined) subset $S(C, D)$ of the strands in $\tau(C, D)$. Let a legal path $P=\cdots\left(\mathbf{C}^{\prime \prime}, D^{\prime \prime}\right) \rightarrow_{x}(\mathbf{C}, D)$ from $(I, J)$ to $(C, D)$ be given, where $\mathbf{C}=\left(c_{1}, c_{2}, \ldots, c_{k}\right)$. With respect to $(\mathbf{C}, D)$, the minimum strand $\left(c_{i}, d\right)$ in $S(C, D)$ is the strand where $i$ is minimal. We define $\iota(P)$ by splitting into two cases.

Case (1): If $|S(C, D)|>0$ and $\left(c_{i}, d\right)$ is the minimal strand, and either

1. $P$ is empty, or

2. $P$ is nonempty and $i \leqslant x$,

then $\iota(P)=P \rightarrow_{i}\left(\mathbf{C}^{\prime}, D^{\prime}\right)$ is the path obtained by concatenating to $P$ the swap $(\mathbf{C}, D) \rightarrow_{i}$ $\left(\mathbf{C}^{\prime}, D^{\prime}\right)$. Note that $\tau\left(\overline{\mathbf{C}}^{\prime}, D^{\prime}\right)$ is still compatible with $(K, L)$ : if $\left(c_{i}, d\right)$ is nested under a strand $\left(c_{s}, d^{\prime}\right)$, then by Lemmas 7 and 8 , we cannot have $\left(c_{s}, d^{\prime}\right) \in S(C, D)$. Thus $S\left(\overline{\mathbf{C}}^{\prime}, D^{\prime}\right)=S(C, D) \backslash\left(c_{i}, d\right)$.

Case (2): If either

1. $|S(C, D)|=0$ (that is, $(C, D)=(I, J))$, or

2. $|S(C, D)|>0$ with $\left(c_{i}, d\right)$ minimal strand, and $P$ is nonempty and $i>x$,

then $\iota(P)$ is obtained from $P$ by removing the last swap, so that $\iota(P)$ now ends at $\left(\mathbf{C}^{\prime \prime}, D^{\prime \prime}\right)$. By Lemmas 7 and 8 again, note that $\tau\left(\mathbf{C}^{\prime \prime}, D^{\prime \prime}\right)$ is compatible with $(I, J)$, and we have $S\left(\overline{\mathbf{C}}^{\prime \prime}, D^{\prime \prime}\right)=S(C, D) \cup\{(p, q)\}$ where $(p, q)$ is the last swap in $P$.

Finally, it is straightforward to verify that $\iota$ is an involution and that $(-1)^{|\iota(P)|}=$ $-(-1)^{|P|}$. 


\section{Schubert varieties and positroid varieties}

\subsection{Schubert varieties}

Let $I \in\left(\begin{array}{c}{[n]} \\ k\end{array}\right)$ be a $k$-element subset of $[n]$. Let $F_{\bullet}=\left\{0=F_{0} \subset F_{1} \subset \cdots F_{n-1} \subset F_{n}=\mathbb{C}^{n}\right\}$ be a flag in $\mathbb{C}^{n}$, so that $\operatorname{dim} F_{i}=i$. The Schubert cell $\dot{\circ}_{I}\left(F_{\bullet}\right)$ is given by

$$
\stackrel{\circ}{X}_{I}\left(F_{\bullet}\right):=\left\{X \in \operatorname{Gr}(k, n) \mid \operatorname{dim}\left(X \cap F_{j}\right)=\#(I \cap[n-j+1, n]) \text { for all } j \in[n]\right\} .
$$

The Schubert variety $X_{I}\left(F_{\bullet}\right)$ is given by

$$
X_{I}\left(F_{\bullet}\right):=\left\{X \in \operatorname{Gr}(k, n) \mid \operatorname{dim}\left(X \cap F_{j}\right) \geqslant \#(I \cap[n-j+1, n]) \text { for all } j \in[n]\right\} .
$$

We have $X_{I}\left(F_{\bullet}\right)=\overline{\dot{O}_{I}\left(F_{\bullet}\right)}$ in the Zariski topology. Also, $X_{[k]}\left(F_{\bullet}\right)=\operatorname{Gr}(k, n)$ and $\operatorname{codim}\left(X_{I}\left(F_{\bullet}\right)\right)=i_{1}+i_{2}+\cdots+i_{k}-(1+2+\cdots+k)$, where $I=\left\{i_{1}, i_{2} \ldots, i_{k}\right\}$. Here and elsewhere, we always mean complex (co)dimension when referring to complex subvarieties.

Let $E_{\bullet}$ be the standard flag defined by $E_{i}=\operatorname{span}\left(e_{n}, e_{n-1}, \ldots, e_{n-i+1}\right)$. Then we set the standard Schubert varieties to be $X_{I}:=X_{I}\left(E_{\bullet}\right)$. Suppose $v_{1}, v_{2}, \ldots, v_{n}$ are the columns of a $k \times n$ matrix (with respect to the basis $e_{1}, e_{2}, \ldots, e_{n}$ ) representing $X \in \operatorname{Gr}(k, n)$. Then the condition $\operatorname{dim}\left(X \cap E_{j}\right)=d$ is equivalent to the condition $\operatorname{dim} \operatorname{span}\left(v_{1}, \ldots, v_{n-j}\right)=$ $k-d$. Thus the Schubert variety $X_{I}\left(E_{\bullet}\right)$ is cut out by rank conditions on initial sequences of columns of $X$.

\subsection{Bounded affine permutations, Grassmann necklaces, and positroids}

A $(k, n)$-bounded affine permutation is a bijection $f: \mathbb{Z} \rightarrow \mathbb{Z}$ satisfying conditions:

1. $f(i+n)=f(i)+n$,

2. $\sum_{i=1}^{n} f(i)=\left(\begin{array}{c}n+1 \\ 2\end{array}\right)+k n$,

3. $i \leqslant f(i) \leqslant i+n$.

The set $\mathcal{B}(k, n)$ of $(k, n)$-bounded affine permutations forms a lower order ideal in the Bruhat order of the affine symmetric group ([KLS13]).

Let $I=\left\{i_{1}<i_{2}<\cdots<i_{k}\right\}$ and $J=\left\{j_{1}<j_{2}<\cdots<j_{k}\right\}$ be two $k$-element subsets of $[n]$. We define a partial order $\leqslant$ on $\left(\begin{array}{c}{[n]} \\ k\end{array}\right)$ by $I \leqslant J$ if $i_{r} \leqslant j_{r}$ for $r=1,2, \ldots, k$. We write $\leqslant_{a}$ for the cyclically rotated ordering $a<_{a} a+1<_{a} \cdots<_{a} n<_{a} 1<_{a} \cdots<_{a} a-1$ on $[n]$. Replacing $\leqslant$ by $\leqslant_{a}$, we also have the cyclically rotated version partial order $I \leqslant a J$ on $\left(\begin{array}{c}{[n]} \\ k\end{array}\right)$.

A $(k, n)$-Grassmann necklace [Pos] is a collection $\mathcal{I}=\left(I_{1}, I_{2}, \ldots, I_{n}\right)$ of $k$-element subsets $I_{i} \in\left(\begin{array}{c}{[n]} \\ k\end{array}\right)$ satisfying the following property for each $a \in[n]$ :

$$
I_{a+1}= \begin{cases}I_{a} & \text { if } a \notin I_{a} \\ I_{a}-\{a\} \cup\left\{a^{\prime}\right\} \text { for some } a^{\prime} \in[n] & \text { if } a \in I_{a} .\end{cases}
$$


There is a partial order on the set of $(k, n)$-Grassmann necklaces, given by $\mathcal{I} \leqslant \mathcal{J}$ if $I_{a} \leqslant a J_{a}$ for all $a=1,2, \ldots, n$.

Given $f \in \mathcal{B}(k, n)$, we define a sequence $\mathcal{I}(f)=\left(I_{1}, I_{2}, \ldots, I_{n}\right)$ of $k$-element subsets by the formula

$$
I_{a}=\{f(b) \mid b<a \text { and } f(b) \geqslant a\} \bmod n
$$

where $\bmod n$ means that we take representatives in $[n]$. For example, let $k=2, n=6$, and $f=[246759]$. Then $\mathcal{I}(f)=(13,23,34,46,16,16)$.

Proposition 9. The map $f \mapsto \mathcal{I}(f)$ is a bijection between $(k, n)$-bounded affine permutations and $(k, n)$-Grassmann necklaces.

The inverse map $\mathcal{I} \mapsto f(\mathcal{I})$ is given as follows. Suppose $a \notin I_{a}$. Then define $f(a)=a$. Suppose $a \in I_{a}$ and $I_{a+1}=I_{a}-\{a\} \cup\left\{a^{\prime}\right\}$. Then define $f(a)=b$ where $b \equiv a^{\prime} \bmod n$ and $a<b \leqslant a+n$.

The Bruhat order on $\left(\begin{array}{c}{[n]} \\ k\end{array}\right)$ is given by $I=\left\{i_{1}<i_{2}<\cdots<i_{k}\right\} \leqslant J=\left\{j_{1}<j_{2}<\cdots<\right.$ $\left.j_{k}\right\}$ if and only if $i_{r} \leqslant j_{r}$ for all $r$. For $I \in\left(\begin{array}{c}{[n]} \\ k\end{array}\right)$, the Schubert matroid $\mathcal{M}_{I}$ is by definition the collection

$$
\mathcal{M}_{I}:=\left\{J \geqslant I \mid J \in\left(\begin{array}{c}
{[n]} \\
k
\end{array}\right)\right\} .
$$

It indexes the set of Plücker coordinates $\Delta_{J}$ that do not vanish on $X_{I}$ [Ram].

Let $\mathcal{I}$ be a $(k, n)$-Grassmann necklace. The positroid $\mathcal{M}(\mathcal{I})$ of $\mathcal{I}$ is the rank $k$ matroid on $n$ elements given by

$$
\mathcal{M}(\mathcal{I}):=\mathcal{M}_{I_{1}} \cap \chi\left(\mathcal{M}_{\chi^{-1}\left(I_{2}\right)}\right) \cap \cdots \cap \chi^{n-1}\left(\mathcal{M}_{\chi^{1-n}\left(I_{n}\right)}\right) .
$$

If $\mathcal{I}=\mathcal{I}(f)$ then we write $\mathcal{M}(f)$ for $\mathcal{M}(\mathcal{I})$.

\subsection{Positroid varieties}

Define the positroid variety $\Pi_{f}$

$$
\Pi_{f}=\Pi_{\mathcal{I}}:=X_{I_{1}} \cap \chi\left(X_{\chi^{-1}\left(I_{2}\right)}\right) \cap \cdots \cap \chi^{n-1}\left(X_{\chi^{1-n}\left(I_{n}\right)}\right)
$$

where $\mathcal{I}=\mathcal{I}(f)$ and the open positroid variety $\stackrel{\circ}{\Pi}_{f}$

$$
\stackrel{\circ}{\Pi}_{f}=\stackrel{\circ}{\Pi}_{\mathcal{I}}:=\stackrel{\circ}{X}_{I_{1}} \cap \chi\left(\stackrel{\circ}{X}_{\chi^{-1}\left(I_{2}\right)}\right) \cap \cdots \cap \chi^{n-1}\left(\stackrel{\circ}{X}_{\chi^{1-n}\left(I_{n}\right)}\right) .
$$

By $[\operatorname{KLS} 13, \mathrm{KLS} 14]$, the restriction map $\Gamma(\operatorname{Gr}(k, n), \mathcal{O}(d)) \rightarrow \Gamma\left(\Pi_{f}, \mathcal{O}(d)\right)$ is surjective, where $\mathcal{O}(1)$ is the line bundle on $\operatorname{Gr}(k, n)$ associated to the Plücker embedding (and in particular, $\Gamma\left(\Pi_{f}, \mathcal{O}(d)\right)=0$ for $\left.d<0\right)$. Thus the homogeneous coordinate ring $R\left(\Pi_{f}\right):=$ $\bigoplus_{d \geqslant 0} \Gamma\left(\Pi_{f}, \mathcal{O}(d)\right)$ of $\Pi_{f}$ is a quotient of the homogenous coordinate ring $R(k, n)$. We write $\mathcal{I}\left(\Pi_{f}\right)$ for the homogeneous ideal of $\Pi_{f}$ and denote by $\hat{\Pi}_{f}:=\operatorname{Spec}\left(R\left(\Pi_{f}\right)\right) \subset \hat{\operatorname{Gr}}(k, n)$ the affine cone over the positroid variety $\Pi_{f}$.

Recall that for $X \in \operatorname{Gr}(k, n)$, the matroid $\mathcal{M}_{X}$ of $X$ is defined as

$$
\mathcal{M}_{X}:=\left\{J \in\left(\begin{array}{c}
{[n]} \\
k
\end{array}\right) \mid \Delta_{J}(X) \neq 0\right\} .
$$

Define $\Pi_{f,>0}:=\stackrel{\circ}{\Pi}_{f} \cap \operatorname{Gr}(k, n)_{\geqslant 0}$. The following result of Oh characterizes the matroids of totally nonnegative points.

Theorem 10 ([Oh]). For any $X \in \Pi_{f,>0}$, we have $\mathcal{M}_{X}=\mathcal{M}(f)$. 


\section{The cyclic Demazure module}

\subsection{Demazure modules and Demazure crystals}

Let $\mathcal{I}\left(X_{I}\right) \subset R(k, n)$ denote the homogeneous ideal of the Schubert variety $X_{I}$ (see Section 4.1) and let $\mathcal{I}\left(X_{I}\right)_{d} \subset R(k, n)_{d}$ denote the degree $d$ component. Let $R\left(X_{I}\right)_{d}=\Gamma\left(X_{I}, \mathcal{O}(d)\right)$ denote the degree $d$ part of the homogeneous coordinate ring of $X_{I}$. The restriction map $\Gamma(\operatorname{Gr}(k, n), \mathcal{O}(d)) \rightarrow \Gamma\left(X_{I}, \mathcal{O}(d)\right)$ is known to be surjective, and thus the space $R\left(X_{I}\right)_{d}$ is naturally a quotient of $R(k, n)_{d}=V\left(d \omega_{k}\right)^{*}$.

For $I \in\left(\begin{array}{c}{[n]} \\ k\end{array}\right)$, we have an extremal weight vector $G\left(T_{I}\right) \in V\left(d \omega_{k}\right)$. The vector $G\left(T_{I}\right)$ spans the weight space of $V\left(d \omega_{k}\right)$ with weight $\alpha=\left(\alpha_{1}, \ldots, \alpha_{n}\right)$ given by $\alpha_{i}=d$ if $i \in I$ and $\alpha_{i}=0$ otherwise. The Demazure module $V_{I}\left(d \omega_{k}\right)$ is defined to be the $B_{-}$-submodule of $V\left(d \omega_{k}\right)$ generated by the vector $G\left(T_{I}\right)$. It is a classical result that $\mathcal{I}\left(X_{I}\right)_{d}$ can be identifed with $V_{I}\left(d \omega_{k}\right)^{\perp} \subset V\left(d \omega_{k}\right)^{*}$ (see for example [Kum, Chapter 8]).

For $I \in\left(\begin{array}{c}{[n]} \\ k\end{array}\right)$, we have a tableau $T_{I} \in B\left(d \omega_{k}\right)$ with all entries in the $r$-th row equal to $i_{r}$, where $I=\left\{i_{1}, i_{2}, \ldots, i_{k}\right\}$. The canonical basis vector $G\left(T_{I}\right)$ is an extremal weight vector of $V\left(d \omega_{k}\right)$. Define the Demazure crystal $B_{I}\left(d \omega_{k}\right) \subset B\left(d \omega_{k}\right)$ to be the subset of $B\left(d \omega_{k}\right)$ obtained by repeatedly applying the operators $\tilde{f}_{1}, \tilde{f}_{2}, \ldots, \tilde{f}_{n-1}$ to $T_{I}$.

The following result is due to Kashiwara [Kas93b].

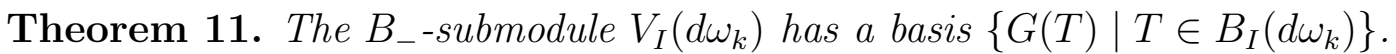

By Theorem 11, we obtain:

Proposition 12. We have

1. $\mathcal{I}\left(X_{I}\right)_{d}=V_{I}\left(d \omega_{k}\right)^{\perp} \subset V\left(d \omega_{k}\right)^{*}=R(k, n)_{d}$ has a basis given by $\{H(T) \mid T \notin$ $\left.B_{I}\left(d \omega_{k}\right)\right\}$.

2. $R\left(X_{I}\right)_{d}$ has a basis given by (the image of) $\left\{H(T) \mid T \in B_{I}\left(d \omega_{k}\right)\right\}$.

Let us give a more explicit description of $B_{I}\left(d \omega_{k}\right)$.

Proposition 13. The set $B_{I}\left(d \omega_{k}\right)$ consists of tableaux $T$ which are entry-wise greater than or equal to $T_{I}$.

Proof. Let $S$ denote the set of tableaux $T \in B\left(d \omega_{k}\right)$ that are entry-wise greater than or equal to $T_{I}$. Since the operators $\tilde{f}_{i}$ increases a single entry of a tableau, it is clear that $B_{I}\left(d \omega_{k}\right)$ is contained in $S$. Also, it is known that the set $S$ indexes a basis for $R\left(X_{I}\right)_{d}$ known as the standard monomial basis, see for example [LaLi]. Thus $\left|B_{I}\left(d \omega_{k}\right)\right|=|S|$, so $B_{I}\left(d \omega_{k}\right)=S$. (Alternatively, we can apply $\tilde{e}_{i}$ to see that $S \subseteq B_{I}\left(d \omega_{k}\right)$.)

\subsection{Cyclic Demazure modules}

Let $f$ be a $(k, n)$-bounded affine permutation. Define $\mathcal{I}\left(\Pi_{f}\right)_{d} \subset R(k, n)_{d}$ by

$$
\mathcal{I}\left(\Pi_{f}\right)_{d}:=\mathcal{I}\left(\Pi_{f}\right) \cap R(k, n)_{d}
$$


to be the degree $d$ homogeneous component of $\mathcal{I}\left(\Pi_{f}\right)$. Since $\mathcal{I}\left(\Pi_{f}\right)$ is a homogeneous ideal, it is spanned by the subspaces $\mathcal{I}\left(\Pi_{f}\right)_{d}$. The aim of this section is to give a representationtheoretic description of $\mathcal{I}\left(\Pi_{f}\right)_{d}$ as a subspace of $R(k, n)_{d} \simeq V\left(d \omega_{k}\right)^{*}$.

Let $f \in \mathcal{B}(k, n)$ have $(k, n)$-Grassmann-necklace $\mathcal{I}(f)=\left(I_{1}, I_{2}, \ldots, I_{n}\right)$. Define the cyclic Demazure crystal $B_{f}\left(d \omega_{k}\right)$ to be intersection

$$
B_{f}\left(d \omega_{k}\right):=B_{I_{1}}\left(d \omega_{k}\right) \cap \chi\left(B_{\chi^{-1}\left(I_{2}\right)}\left(d \omega_{k}\right)\right) \cap \cdots \cap \chi^{n-1}\left(B_{\chi^{1-n}\left(I_{n}\right)}\left(d \omega_{k}\right)\right) .
$$

If we identify $B\left(\omega_{k}\right)$ with the set $\left(\begin{array}{c}{[n]} \\ k\end{array}\right)$ of $k$-element subsets of $[n]$, then $B_{f}\left(\omega_{k}\right)$ is simply the positroid $\mathcal{M}(f)(9)$. Also, define the cyclic Demazure module $V_{f}\left(d \omega_{k}\right)$ to be intersection

$$
V_{f}\left(d \omega_{k}\right):=V_{I_{1}}\left(d \omega_{k}\right) \cap \chi\left(V_{\chi^{-1}\left(I_{2}\right)}\left(d \omega_{k}\right)\right) \cap \cdots \cap \chi^{n-1}\left(V_{\chi^{1-n}\left(I_{n}\right)}\left(d \omega_{k}\right)\right) .
$$

Let $R\left(\Pi_{f}\right)$ denote the homogeneous coordinate ring of the positroid variety $\Pi_{f}$.

Theorem 14. The subspace $V_{f}\left(d \omega_{k}\right)$ has a basis $\left\{G(T) \mid T \in B_{f}\left(d \omega_{k}\right)\right\}$.

\section{Theorem 15.}

(i) $\mathcal{I}\left(\Pi_{f}\right)_{d}$ is isomorphic to $V_{f}\left(d \omega_{k}\right)^{\perp}$ and has a basis given by $\left\{H(T) \mid T \notin B_{f}\left(d \omega_{k}\right)\right\}$.

(ii) $R\left(\Pi_{f}\right)_{d}$ has a basis given by the images of $\left\{H(T) \mid T \in B_{f}\left(d \omega_{k}\right)\right\}$.

Theorem 15 reduces in the case $d=1$ to Theorem 10 .

Corollary 16. For $f \in \mathcal{B}(k, n)$ and $d \geqslant 1$, the cyclic Demazure crystal $B_{f}\left(d \omega_{k}\right)$ is nonempty.

Indeed, for any $a$, the function $\Delta_{I_{a}}$ is non-zero on $\hat{\Pi}_{f}$, and so is $\Delta_{I_{a}}^{d}$. Thus, $B_{f}\left(d \omega_{k}\right)$ contains the tableaux $T_{I_{1}}, T_{I_{2}}, \ldots, T_{I_{n}}$.

Remark 17. It follows from Theorem 15 that the vectors $H(T) \in V\left(d \omega_{k}\right)^{*}$ that do not restrict to identically zero on $\Pi_{f}$ form a basis for $R\left(\Pi_{f}\right)_{d}$. This is not the case for the standard monomial basis (cf. [LaLi]).

Example 18. Suppose $k=1$. In this case $B_{I}\left(d \omega_{1}\right)$ is the set of one-row tableaux (of length $d$ ) with entries in $1,2, \ldots, i$, where $I=\{i\}$. By choosing the $(1, n)$-Grassmann necklace appropriately, $B_{f}\left(\omega_{1}\right)$ can be arranged to be any subset of $\{1,2, \ldots, n\}$. For example, if $n=4,\left(I_{1}, I_{2}, I_{3}, I_{4}\right)=(1,3,3,1)$ gives $B_{f}\left(\omega_{1}\right)=\{1,3\}$. The set $B_{f}\left(d \omega_{1}\right)$ is simply the set of one-row tableaux with entries in $B_{f}\left(\omega_{1}\right)$.

Example 19. Take $k=2$ and $n=4$. Let us consider the positroid variety $\Pi_{f}$ where $f=[2547] \in \mathcal{B}(2,4)$. The Grassmann necklace is $\mathcal{I}(f)=(13,23,13,41)$. The set $B_{f}\left(2 \omega_{2}\right)$ is given by the set of tableaux

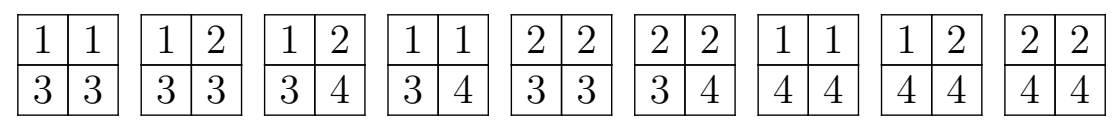


Example 20. Consider $k=2$ and $n=5$. Let us consider the positroid variety $\Pi_{f}$ where $f=[63547] \in \mathcal{B}(2,5)$ and compute $B_{f}\left(2 \omega_{2}\right)$. The Grassmann necklace is $\mathcal{I}(f)=$ $(12,12,13,15,15)$. Since $B_{12}\left(2 \omega_{2}\right)=B\left(2 \omega_{2}\right)$, we have

$$
B_{f}\left(2 \omega_{2}\right)=\chi\left(B_{15}\left(2 \omega_{2}\right)\right) \cap \chi^{2}\left(B_{14}\left(2 \omega_{2}\right)\right) \cap \chi^{3}\left(B_{23}\left(2 \omega_{2}\right)\right) .
$$

The set $B_{15}\left(2 \omega_{2}\right)$ consists of all tableaux of the form \begin{tabular}{|l|l|}
\hline$a$ & $b$ \\
\hline 5 & 5 \\
\hline
\end{tabular} with $1 \leqslant a \leqslant b \leqslant 4$ and thus $\chi\left(B_{15}\left(2 \omega_{2}\right)\right)$ consists of all tableaux of the form \begin{tabular}{|l|l|}
\hline 1 & 1 \\
\hline$a$ & $b$
\end{tabular} with $2 \leqslant a \leqslant b \leqslant 5$. In particular, every tableau in $B_{f}\left(2 \omega_{2}\right)$ has exactly two 1-s. Intersecting with $\chi^{2}\left(B_{14}\left(2 \omega_{2}\right)\right)$ imposes no additional restriction. On the other hand, looking at tableaux in $B_{23}\left(2 \omega_{2}\right)$ with two 3 -s, we get the six tableaux

\begin{tabular}{|c|c|c|c|c|c|c|c|c|c|c|c|}
\hline 2 & 2 & 2 & 3 & 2 & 3 & 3 & 3 & & 3 & 3 & 3 \\
\hline 3 & 3 & 3 & 4 & 3 & 5 & 4 & 4 & 4 & 5 & 5 & 5 \\
\hline
\end{tabular}

and thus $B_{f}\left(2 \omega_{2}\right)$ consists of the tableaux

\begin{tabular}{|c|c|c|c|c|c|c|c|c|c|c|c|}
\hline 1 & 1 & 1 & 1 & 1 & 1 & 1 & 1 & 1 & 1 & 1 & 1 \\
\hline 5 & 5 & 2 & 5 & 3 & 5 & 2 & 2 & 2 & 3 & 3 & 3 \\
\hline
\end{tabular}

Next we give an example of a Schubert variety whose ideal does not have a basis given by a subset of the dual canonical basis.

Example 21. Let $X \subset \operatorname{Gr}(2,4)$ be given by the single equation $\left\{\Delta_{13}=0\right\}$. This is a permutation of a standard Schubert variety that is not a positroid variety. Then the degree two part of $\mathcal{I}(X)$ has a one-dimensional weight space for the weight $(1,1,1,1)$. It is spanned by the vector $\Delta_{13} \Delta_{24}$. This vector is a sum of two elements of the dual canonical basis by Theorem 3 .

\subsection{Proof of Theorem 14}

By Theorem 11, $V_{\chi^{1-a}\left(I_{a}\right)}\left(d \omega_{k}\right)$ has basis $\left\{G(T) \mid T \in B_{\chi^{1-a}\left(I_{a}\right)}\left(d \omega_{k}\right)\right\}$. Thus by Theorem $1($ iv $)$, the rotation $\chi^{a-1}\left(V_{\chi^{1-a}\left(I_{a}\right)}\left(d \omega_{k}\right)\right)$ has basis given by

$$
\left\{G(T) \mid T \in \chi^{a-1} B_{\chi^{1-a}\left(I_{a}\right)}\left(d \omega_{k}\right)\right\} .
$$

It follows that the intersection (11) has basis $\left\{G(T) \mid T \in B_{f}\left(d \omega_{k}\right)\right\}$, establishing Theorem 14.

\subsection{Proof of Theorem 15}

Our proof of Theorem 15 relies on the following result proved jointly with Knutson and Speyer [KLS13, KLS14]. It states that the intersection in (10) is reduced, so the equality in (10) holds as schemes. 
Proposition 22. The homogeneous ideal $\mathcal{I}_{f}$ of a positroid variety is given by

$$
\mathcal{I}\left(\Pi_{f}\right)=\mathcal{I}\left(X_{I_{1}}\right)+\chi\left(\mathcal{I}\left(X_{\chi^{-1}\left(I_{2}\right)}\right)\right)+\cdots+\chi^{n-1}\left(\mathcal{I}\left(X_{\chi^{1-n}\left(I_{n}\right)}\right)\right) .
$$

Since $(A \cap B)^{\perp}=A^{\perp}+B^{\perp}$, combining with Proposition 12, we deduce that

$$
\mathcal{I}\left(\Pi_{f}\right)_{d}=\left(V_{I_{1}}\left(d \omega_{k}\right) \cap \chi\left(V_{\chi^{-1}\left(I_{2}\right)}\left(d \omega_{k}\right)\right) \cap \cdots \cap \chi^{n-1}\left(V_{\chi^{1-n}\left(I_{n}\right)}\left(d \omega_{k}\right)\right)\right)^{\perp}=V_{f}\left(d \omega_{k}\right)^{\perp} .
$$

Combining with Theorem 11, we obtain Theorem 15(i). Theorem 15(ii) follows from the isomorphism of vector spaces $R\left(\Pi_{f}\right)_{d} \cong R(k, n)_{d} / \mathcal{I}\left(\Pi_{f}\right)_{d}$.

\subsection{Positivity}

Theorem 23. For $f \in \mathcal{B}(k, n)$ and $T \in B\left(d \omega_{k}\right)$, if $H(T)$ is not identically zero on $\Pi_{f}$, then it takes strictly positive values everywhere on $\Pi_{f,>0}$.

Proof. Fix $f \in \mathcal{B}(k, n)$. By [Lam16, Section 7], the totally nonnegative cell $\Pi_{f,>0} \cong \mathbb{R}_{>0}^{d}$ has a parametrization of the following form:

$$
\mathbb{R}_{>0}^{d} \ni\left(t_{1}, t_{2}, \ldots, t_{d}\right) \mapsto x_{i_{1}}\left(t_{1}\right) \cdots x_{i_{d}}\left(t_{d}\right) \cdot e_{I} \in \Pi_{f,>0}
$$

where $e_{I} \in \operatorname{Gr}(k, n)_{\geqslant 0}$ for $I=\left\{i_{1}, \ldots, i_{k}\right\} \in\left(\begin{array}{c}{[n]} \\ k\end{array}\right)$ denotes the point

$$
e_{I}=\operatorname{span}\left(e_{i_{1}}, e_{i_{2}}, \ldots, e_{i_{k}}\right) \in \operatorname{Gr}(k, n)_{\geqslant 0}
$$

and $x_{i}(a)=\exp \left(a e_{i, i+1}\right) \in \mathrm{GL}(n)$ is the one parameter subgroup associated to the Chevalley generator for $i=1,2, \ldots, n-1$. For $i=0$, we define $x_{0}(a)$ by conjugating $x_{1}(a)$ by $\chi$.

Fix a lift of $e_{I}$ to $\hat{\operatorname{Gr}}(k, n)$. Then the value of the dual canonical basis element $H(T) \in$ $V\left(d \omega_{k}\right)^{*}$ on the point $X=x_{i_{1}}\left(t_{1}\right) \cdots x_{i_{d}}\left(t_{d}\right) \cdot e_{I} \in \hat{\operatorname{Gr}}(k, n)$ is given by

$$
\left\langle H(T), x_{i_{1}}\left(t_{1}\right) \cdots x_{i_{d}}\left(t_{d}\right) \cdot G\left(T_{I}\right)\right\rangle
$$

where $\langle\cdot, \cdot\rangle$ denotes the natural pairing between $V\left(d \omega_{k}\right)^{*}$ and $V\left(d \omega_{k}\right)$, and $G\left(T_{I}\right)$ is the canonical basis element of extremal weight indexed by $T_{I}$.

Thus it suffices to show that for any $T \in B\left(d \omega_{k}\right)$, the coefficient of $G(T)$ in the vector $x_{i_{1}}\left(t_{1}\right) \cdots x_{i_{d}}\left(t_{d}\right) \cdot G\left(T_{I}\right)$ is equal to a (possibly zero) polynomial in $t_{1}, t_{2}, \ldots, t_{d}$ with nonnegative coefficients. For $i=1,2, \ldots, n-1$, it follows from the proof of [Lus94, Proposition 3.2] that the matrix coefficients of $x_{i}(a)$ on the canonical basis of $V\left(d \omega_{k}\right)$ are polynomials in $t_{1}, t_{2}, \ldots, t_{d}$ with nonnegative coefficients. By Theorem 1(iv), the same holds for $i=0$. The claim follows.

\section{$6 \quad$ Future directions}

\subsection{The character of the cyclic Demazure module}

The character of highest weight representation $V\left(d \omega_{k}\right)$ is given by the celebrated Weyl character formula. The character of the Demazure module $V_{I}\left(d \omega_{k}\right)$ is given by the Demazure character formula [Dem, And]. 
Problem 24. Find a formula for the character of $V_{f}\left(d \omega_{k}\right)$. Equivalently, compute the weight generating function of $B_{f}\left(d \omega_{k}\right)$.

For the bounded affine permutation $f=[2547]$ of Example 19, we have $\operatorname{ch}\left(V_{f}\left(2 \omega_{2}\right)\right)=x_{1}^{2} x_{3}^{2}+x_{1}^{2} x_{3} x_{4}+x_{1}^{2} x_{4}^{2}+x_{1} x_{2} x_{3}^{2}+x_{1} x_{2} x_{3} x_{4}+x_{1} x_{2} x_{4}^{2}+x_{2}^{2} x_{3}^{2}+x_{2}^{2} x_{3} x_{4}+x_{2}^{2} x_{4}^{2}$ and for $f=[63547]$ of Example 20, we have

$$
\operatorname{ch}\left(V_{f}\left(2 \omega_{2}\right)\right)=x_{1}^{2}\left(x_{2}^{2}+x_{2} x_{3}+x_{2} x_{5}+x_{3}^{2}+x_{3} x_{5}+x_{5}^{2}\right) .
$$

\subsection{Quantization}

Quantum versions of Grassmannians and Schubert varieties have been studied by many authors, see for example [LeRi]. In that setting, positroid varieties correspond to certain torus-invariant prime ideals, classified in [MéCa, Yak].

Problem 25. Find the quantum version of the cyclic Demazure module, and quantum versions of Theorems 14 and 15 .

Note however that the cyclic symmetry acts on the quantum Grassmannian in a more subtle way than it does on the Grassmannian [LaLe11].

\subsection{Higher degree matroids}

Definition 26. For an integer $d \geqslant 1$, and $X \in \operatorname{Gr}(k, n)$, define the degree $d$ canonical basis matroid

$$
\mathcal{M}_{X, d}:=\left\{T \in B\left(d \omega_{k}\right) \mid H(T)(X) \neq 0\right\} .
$$

By Theorem 1(i), for $d=1, \mathcal{M}_{X, 1}$ is the usual matroid of $X$. By Theorem 23, the degree $d$ canonical basis matroids $\mathcal{M}_{X, d}$ of a point $X \in \operatorname{Gr}(k, n)_{\geqslant 0}$ is completely determined by the usual positroid $\mathcal{M}_{X}$. Thus for any $d$, there is a natural bijection between $\mathcal{B}(k, n)$ and the set of degree $d$ positroids, sending $f \in \mathcal{B}(k, n)$ to $B_{f}\left(d \omega_{k}\right)$.

Problem 27. Find axioms for degree $d$ canonical basis matroids.

\subsection{Projective geometry interpretation of dual canonical basis}

Let $X$ be a $k \times n$ matrix representing a point in $\operatorname{Gr}(k, n)$. We assume that all columns of $X$ are non-zero and think of $X$ as a collection $p_{1}, p_{2}, \ldots, p_{n}$ of points in $\mathbb{P}^{k-1}$. The vanishing of $\Delta_{i_{1}, \ldots, i_{k}}(X)$ is equivalent to the geometric statement that $p_{i_{1}}, \ldots, p_{i_{k}}$ do not span the whole of $\mathbb{P}^{k-1}$.

The Temperley-Lieb invariants $\Delta_{(\tau, T)}$ can be interpreted as tensor invariants [FLL, Appendix], and thereby we obtain an interpretation of degree two matroids in geometric terms. For example, let $k=3$ and $n=6$. If $\tau=\{(1,6),(2,5),(3,4)\}$, then $\Delta_{(\tau, \emptyset)}=$ $\Delta_{123} \Delta_{456}$, which vanishes if and only if either $p_{1}, p_{2}, p_{3}$ are colinear or $p_{4}, p_{5}, p_{6}$ are colinear. If $\tau=\{(1,2),(3,4),(5,6)\}$, then $\Delta_{(\tau, \emptyset)}$ vanishes if the lines $\overline{23}, \overline{45}$, and $\overline{16}$ have a common intersection point. (If any of these pairs, say $p_{2}$ and $p_{3}$, do not span a line, then $\Delta_{(\tau, \emptyset)}$ also vanishes.) 
Problem 28. Give an interpretation of the vanishing of the function $H(T), T \in B\left(d \omega_{k}\right)$ in projective geometry terms.

See also [FoPy, BHL, Lam16] for related work.

\subsection{Two maps on $R(k, n)$}

We have a map

$$
\wedge: \Lambda^{\ell}\left(\mathbb{C}^{n}\right) \otimes \Lambda^{k}\left(\mathbb{C}^{n}\right) \rightarrow \Lambda^{k+\ell}\left(\mathbb{C}^{n}\right)
$$

induced by

$$
\left(e_{i_{1}} \wedge e_{i_{2}} \wedge \cdots \wedge e_{i_{\ell}}\right) \otimes\left(e_{j_{1}} \wedge e_{j_{2}} \wedge \cdots \wedge e_{j_{k}}\right) \longmapsto e_{i_{1}} \wedge \cdots \wedge e_{i_{\ell}} \wedge e_{j_{1}} \wedge \cdots \wedge e_{j_{k}}
$$

This is a map of GL(n)-representations, and up to scalar, it is the unique such map, since the multiplicity of $\Lambda^{k+\ell}\left(\mathbb{C}^{n}\right)$ in $\Lambda^{\ell}\left(\mathbb{C}^{n}\right) \otimes \Lambda^{k}\left(\mathbb{C}^{n}\right)$ is equal to one.

Similarly, by [Ste, Theorem 3.1], the GL(n)-representation $V\left(d \omega_{\ell}\right) \otimes V\left(d \omega_{k}\right)$ is multiplicity free, and in particular, the irreducible representation $V\left(d \omega_{k+\ell}\right)$ appears with multiplicity one. We thus have a canonical (up to scalar) surjective map

$$
\kappa_{k, \ell}^{d}: V\left(d \omega_{\ell}\right) \otimes V\left(d \omega_{k}\right) \rightarrow V\left(d \omega_{k+\ell}\right)
$$

of $\operatorname{GL}(n)$-representations.

Problem 29. Give an explicit combinatorial formula for the expansion of $\kappa_{k, \ell}^{d}(G(T) \otimes$ $\left.G\left(T^{\prime}\right)\right)$ in the canonical basis.

We have obtained an explicit combinatorial solution to Problem 29 for Temperley-Lieb invariants, which we hope to explain elsewhere.

Remark 30. Let $\phi_{k, \ell}: \operatorname{Gr}(k, n) \times \operatorname{Gr}(\ell, n) \rightarrow \operatorname{Gr}(k+\ell, n)$ be the (rational) direct sum map [Lam16], sending $(V, W)$ to $V \bigoplus W$. It is not difficult to see that the map $\kappa_{k, \ell}^{d}$ can be chosen to be dual to the map $R(k+\ell, n)_{d} \rightarrow R(\ell, n)_{d} \otimes R(k, n)_{d}$ induced by $\phi_{k, \ell}$. The study of $\phi_{k, \ell}$ was one of the main motivations for the present work, and we refer the reader to [Lam16] for further details.

Remark 31. An alternating formula for $\kappa_{k, \ell}^{d}\left(G(T) \otimes G\left(T^{\prime}\right)\right)$ can be computed in terms of Kazhdan-Lusztig polynomials, for example by work of Brundan [Bru].

Similarly, there is (up to scalar) a unique non-trival GL( $n)$ homomorphism

$$
\eta_{k}^{d, d^{\prime}}: V\left(\left(d+d^{\prime}\right) \omega_{k}\right) \rightarrow V\left(d \omega_{\ell}\right) \otimes V\left(d^{\prime} \omega_{k}\right)
$$

This map is dual to the natural multiplication map $R(k, n)_{d} \otimes R(k, n)_{d^{\prime}} \rightarrow R(k, n)_{d+d^{\prime}}$ of the homogeneous coordinate ring.

Problem 32. Give an explicit combinatorial formula for the expansion of $\eta_{k}^{d, d^{\prime}}(G(T))$ in the canonical basis. 


\section{References}

[And] H.H. Andersen. Schubert varieties and Demazure's character formula. Invent. Math. 79 (1985), no. 3, 611-618.

[BHL] Y. Bai, S. He, and T. Lam. The amplituhedron and the one-loop Grassmannian measure. J. High Energy Phys. 2016, no. 1, 112, front matter+41 pp.

[Bru] J. Brundan. Dual canonical bases and Kazhdan-Lusztig polynomials. J. Algebra 306 (2006), no. 1, 17-46.

[CWZ] S.-J. Cheng, W. Wang, and R.B. Zhang. Super duality and Kazhdan-Lusztig polynomials, Trans. AMS 360 (2008), 5883-5924.

[Dem] M. Demazure. Désingularisation des variétés de Schubert généralisées. (French) Collection of articles dedicated to Henri Cartan on the occasion of his 70th birthday, I. Ann. Sci. École Norm. Sup. (4) 7 (1974), 53-88.

[Du92] J. Du. Canonical bases for irreducible representations of quantum $\mathrm{GL}_{n}$. Bull. London Math. Soc. 24 (1992), no. 4, 325-334.

[Du95] J. Du. Canonical bases for irreducible representations of quantum GL $_{n}$. II. J. London Math. Soc. (2) 51 (1995), no. 3, 461-470.

[FoPy] S. Fomin and P. Pylyavskyy. Tensor diagrams and cluster algebras. Adv. Math. 300 (2016), 717-787.

[FLL] C. Fraser, T. Lam, and I. Le. From dimers to webs. Trans. Amer. Math. Soc. 371 (2019), 6087-6124.

[HKOTT] G. Hatayama, A. Kuniba, M. Okado, T. Takagi, and Z. Tsuboi. Paths, crystals and fermionic formulae. MathPhys odyssey, 2001, 205-272, Prog. Math. Phys., 23, Birkhäuser Boston, Boston, MA, 2002.

[Kas93a] M. Kashiwara. Global crystal bases of quantum groups. Duke Math. J. 69 (1993), no. $2,455-485$.

[Kas93b] M. Kashiwara. The crystal base and Littelmann's refined Demazure character formula. Duke Math. J. 71 (1993), no. 3, 839-858.

[KLS13] A. Knutson, T. Lam, and D. Speyer. Positroid varieties: juggling and geometry. Compos. Math. 149 (2013), no. 10, 1710-1752.

[KLS14] A. Knutson, T. Lam, and D. Speyer. Projections of Richardson varieties. J. Reine Angew. Math. 687 (2014), 133-157.

[Kum] S. Kumar. Kac-Moody groups, their flag varieties and representation theory. Progress in Mathematics, 204. Birkhäuser Boston, Inc., Boston, MA, 2002. xvi+606 pp.

[LaLi] V. Lakshmibai and P. Littelmann. Richardson varieties and equivariant K-theory. Special issue celebrating the 80th birthday of Robert Steinberg. J. Algebra 260 (2003), no. 1, 230-260.

[Lam14] T. Lam. Dimers, webs, and positroids. J. Lond. Math. Soc. (2) 92 (2015), no. 3, 633-656. 
[Lam16] T. Lam. Totally nonnegative Grassmannian and Grassmann polytopes. Current developments in mathematics 2014, 51-152, Int. Press, Somerville, MA, 2016.

[LaLe11] S. Launois and T.H. Lenagan. Twisting the quantum Grassmannian. Proc. Amer. Math. Soc. 139 (2011), no. 1, 99-110.

[LeRi] T.H. Lenagan and L. Rigal. Quantum analogues of Schubert varieties in the Grassmannian. Glasg. Math. J. 50 (2008), no. 1, 55-70.

[Lus90] G. Lusztig. Canonical bases arising from quantized enveloping algebras. J. Amer. Math. Soc. 3 (1990), no. 2, 447-498.

[Lus94] G. Lusztig. Total positivity in reductive groups. Lie theory and geometry, 531568, Progr. Math., 123, Birkhäuser Boston, Boston, MA, 1994.

[MéCa] A. Mériaux and G. Cauchon. Admissible diagrams in $U_{q}^{w}(\mathfrak{g})$ and combinatoric properties of Weyl groups, Represent. Theory 14 (2010), 645-687.

[Oh] S. Oh. Positroids and Schubert matroids, Journal of Combinatorial Theory, Series A 118, (2011), 2426-2435.

[Pos] A. Postnikov. Total positivity, Grassmannians, and networks, preprint; http:// math.mit.edu/ apost/papers/tpgrass.pdf.

[Ram] A. Ramanathan. Equations defining Schubert varieties and Frobenius splitting of diagonals, Publ. Math. IHES 65 (1987), 61-90.

[Rho] B. Rhoades. Cyclic sieving, promotion, and representation theory. Journal of Combinatorial Theory, Series A, 117 (1), (2010), 38-76.

[Ska] M. Skandera. On the dual canonical and Kazhdan-Lusztig bases and 3412, 4231avoiding permutations. J. Pure App. Algebra, 212, (2008) 1086-1104.

[Shi] M. Shimozono. Affine type A crystal structure on tensor products of rectangles, Demazure characters, and nilpotent varieties. J. Algebraic Combin. 15 (2002), no. 2, 151-187.

[Ste] J. R. Stembridge. Multiplicity-free products and restrictions of Weyl characters. Represent. Theory 7 (2003), 404-439.

[Yak] M. Yakimov. Invariant prime ideals in quantizations of nilpotent Lie algebras. Proc. London Math. Soc. (3) 101 (2010), no. 2, 454-476. 